\title{
TWO-DIMENSIONAL NUMERICAL SIMULATION OF THE COMBINED HEAT TRANSFER IN CHANNEL FLOW
}

\author{
Ali Yari1, Siamak Hosseinzadeh ${ }^{2 *}$, Meghdad Rahimi Galogahi ${ }^{3}$ \\ ${ }^{1}$ Department of Mechanical Engineering, Shiraz University, Shiraz, Iran \\ Ali.Yari.Engineer@gmaail.com \\ ${ }^{2 *}$ Department of Mechanical Engineering, West Tehran Branch, Islamic Azad \\ University, Tehran, Iran \\ Hoseinzadeh.siamak@gmail.com \\ ${ }^{3}$ Department of Mechanical Engineering, Islamic Azad University, Behshahr Branch, \\ Mazandaran, Iran \\ rahimi meghdadeyahoo.com
}

\begin{abstract}
A numerical investigation was conducted to analyze the flow field and heat transfer characteristics in a vertical channel with radiation and blowing from the wall. Hydrodynamic behaviour and heat transfer results are obtained by the solution of the complete Navier-Stokes and energy equations using a control volume finite element method. Turbulent flow with "Low Reynolds Spalart-Allmaras Turbulence Model" and radiation with "Discrete Transfer Radiation Method" had been modeled. In order to have a complete survey, this article has a wide range of study in different domains including velocity profiles at different locations, turbulent viscosity, shear stress, suctioned mass flow rate in different magnitude of the input Rayleigh number, blowing Reynolds number, radiation parameter, Prandtl number, the ratio of length to width and also ratio of opening thickness to width of the channel. In addition, effects of variation in any of the above non-dimensional numbers on parameters of the flow are clearly illustrated. At the end resultants had been compared with experimental data which demonstrated that in the present study, results have a great accuracy, relative errors are very small and the curve portraits are in a great agreement with real experiments.
\end{abstract}

\section{KEYWORDS}

Turbulent free convection, Shear stress, Radiation, Blowing

\section{INTRODUCTION}

Investigation on boundary layer of free turbulent convection along a vertical plate is really vital not only in terms of heat transfer mechanism, but also in clarifying the turbulent movement phenomenon. Tsuji et al $[1,2]$ measured the average and turbulent values of velocity and temperature profiles, as well as heat transfer rate and wall shear stress for boundary layer of turbulent free convection flow of the air. His results revealed that the boundary layer of free convection flow has a special turbulent feature that has been fairly seen in other boundary layer of other turbulent flows. On the other hand, the effect of fluid blowing from the surface can be an interesting issue that has been studied for a long time [3]. In the boundary layer, blowing causes an increase in thickness of the boundary level and makes the flow turbulent. The effect of blowing and suction on the boundary layers in free convection flow had been proposed by Eichhorn [4] in which the equations for a vertical wall with site function's temperature were solved using a similarity method. The results showed that blowing and suction in laminar flow has little effect on coefficient of friction. Blowing through a flat plate or a cylinder has been studied both experimentally [5,6] and numerically [7-12] for many years but also more 
recently. Similar articles have investigated the issue with other methods (more general modes) to evaluate the effect of blowing and suction (about heat transfer inside the channel) on the parameters of the wall shear stresses [13-15]. In many modern systems, such as compact heat exchangers and electronic parts, the mutual effects of turbulent free convection and radiation are considered on each other. Cheng and Muller [16], in a numerical and experimental investigation, examined turbulent free convection flow of air along with thermal radiation within a three-dimensional rectangular channel with a constant hot wall temperature, this study considered a high Reynolds number. They studied the impact of temperature and emission coefficients changes on the velocity and temperature profiles, intake air mass flow rate from end of the channel and heat transfer. Dong and Lu [17] applied LES to a thermally stratified turbulent channel and studied the effects of temperature oscillations on the lower wall. Wang and Pletcher [18] investigated the influence of fluid properties variations in a turbulent channel flow with significant heat transfer. Murata and Mochizuki [19, 20] studied turbulent heat transfer in a two-pass square channel. Lee et al. [21] carried out simulations in a vertical channel with several temperature differences to study the coupling of fluid properties variations and gravity. Chatelain et al. [22] investigated numerical schemes for LES of heat transfer. Recently, problems including heat transfer, radiation, and blowing has been more considered. Abd ElAziz's [23] study represents the effects of thermal-diffusion on the free convection of heat and mass transfer over a permeable plate with suction-blowing, radiation and magnetic field.

The most important point about present study is the fact that this paper considers most of fluid flow parameters including velocity, temperature, wall shear stresses, turbulent viscosity and suctioned mass flow rate from inputs while flow occurs in vertical channels with three parameters free convection, the radiation and blowing in turbulent flow.

\section{Physical Model}

This model consists of a channel with two parallel plates, where height is called $\mathrm{H}$ and width is called L. The left wall is Isolated and the right wall is grooved with constant temperature. Blowing hot gas is done with the same as wall's temperature and uniform velocity from grooved wall, with increase in width and distance. Fluid flow in the channel is created due to the buoyancy force. In the entrance of the channel, fluid is entered with ambient temperature and the atmospheric pressure and hot exhaust gas is discharged to the atmosphere. It is assumed that all surfaces are gray, radiation properties are independent of the wavelength, the radiation coefficient is equal with the absorption coefficient and the fluid is non-participants in the process of radiation.

\section{Physical Model}

All the fluid properties are assumed to be constant, except density terms of free convection in the momentum equations in which had been modeled assuming the Boussinesq. It is assumed that all surfaces are gray, radiation properties are independent of the wavelength, the radiation coefficient is equal with the absorption coefficient and the fluid is non-participants in the process of radiation. The governing equations for steady two-dimensional state of noncompressible flow, using the conservation laws of mass, momentum and energy can thus be written as:

$$
\begin{aligned}
& \left(\rho V_{i}\right)_{, i}=0 \\
& \rho V_{j} \frac{\partial V_{i}}{\partial X_{j}}=\mu \frac{\partial}{\partial X_{j}}\left(\frac{\partial V_{i}}{\partial X_{j}}\right)+g_{i} \beta\left(T-T_{\infty}\right)
\end{aligned}
$$


$\rho C_{p} V_{j} \frac{\partial T}{\partial X_{j}}=-\beta T V_{j} \frac{\partial P}{\partial X_{j}}+\mu\left(\frac{\partial V_{i}}{\partial X_{j}}+\frac{\partial V_{j}}{\partial X_{i}}\right) \frac{\partial V_{i}}{\partial X_{j}}+k_{t h} \frac{\partial}{\partial X_{i}}\left(\frac{\partial T}{\partial X_{i}}\right)$

Where all parameters have their conventional definitions. The right wall has Constant temperature and the left wall is isolated, thus radiation flux and flux transport in this boundary are equal ( $q_{c}=q_{r}$ ). By using Discrete Transfer Radiation Method and "ray tracing" technique, a system of radiation equations are derived, and after solving conservation equations, the flow field and temperature can be determined.

\section{Discrete Transfer Radiation Method and Ray tracking TECHNIQUE}

The main assumption in this model is the fact that amount of radiation which each element leaves in a certain range of space angles can be estimated by a single beam. In every radiation area, the radiation which is released in discrete values for polar angle $(\theta)$ and horizontal $(\Phi)$ and each beam can be followed to determine the element which is reached and absorbed.

\section{THE STUDY OF NETWORK AND VALIDITY OF RESULTS}

The numerical results for free convective heat transfer in a vertical channel with asymmetric heating and non-blowing wall had been compared with Cheng and Muller's [2] experimental data. In this survey, the channel width ratio $(\mathrm{H} / \mathrm{L})$ is 16 , and the ratio of the whole diameter and gap width with the channel width $(\mathrm{d} / \mathrm{L})$ is 0.008 , respectively radiation ratio is 0.9 and the Rayleigh number equals to $6.5 \times 108$. The act of gridding computational range was according to non-uniform gridding of Form $\mathrm{H}$ in Fig. [1]. Due to ensure the independence of numerical results relative to the grid, the three meshes with $80,000,120,000$, and 200,000 cells were used to compute. The results of velocity and temperature profiles were similar in all three output channels with high accuracy Fig. [2], and show on average, $12 \%$ and $18 \%$, respectively, different with the experimental data. This is an acceptable accuracy for a numerical solution and confirms the usage of Spalart Allmaras for turbulent flow model. In order to existent of y+ constraint, it is not possible to utilize grids with less than 80000 computational cells. For all presented results, the mesh density or a better one was utilized.

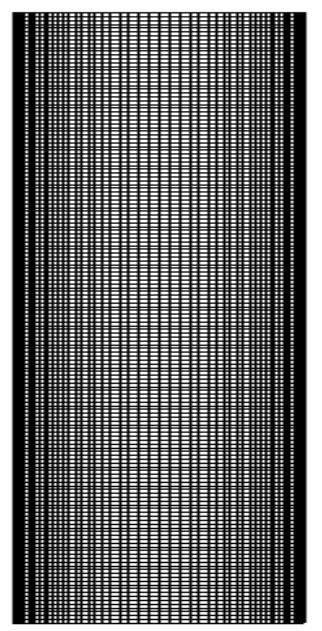

Figure 1: Non-uniform grid for computing zone 


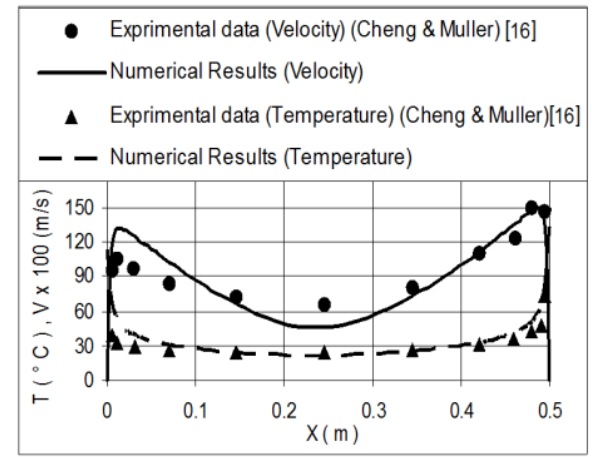

Figure 2. Velocity and temperature profiles in the outlet channel without blowing

$$
\mathrm{H} / \mathrm{L}=16, \mathrm{~d} / \mathrm{L}=0.0008, \varepsilon=0.9, \mathrm{RaH}=6.5^{*} 10^{\wedge} 8
$$

\section{NUMERICAL RESULTS AND DISCUSSION}

Flow in geometry are considered for the function of six independent dimensionless parameters which include the Rayleigh number for vertical plate's height, $\mathrm{Ra}_{\mathrm{H}}$, blowing Reynolds, $\mathrm{Re}_{\mathrm{B}}$, radiation parameters (number of Stephen ), Stef, Prandtl number, Pr, the ratio of height to width of the channel, $H / L$, and the ratio of tracks thickness to channel width, $d / L$. This article presents resultants of the impacts in three parameters: Rayleigh number for vertical hot plate, blowing Reynolds number and radiation parameter (Stephen number). These three variable parameters are respectively defined as:

$$
R a_{H}=\frac{g \beta \Delta T H^{3}}{\alpha v} \quad, \quad \operatorname{Re}_{B}=\frac{\rho V_{B} d}{\mu} \quad, \quad S t e f=\frac{4 \sigma \cdot \varepsilon \cdot T_{1}^{3} \cdot L}{k}
$$

It is important to know that alteration in any of these variables will affect the flow parameters. Thus the problem for different values of the dimensionless numbers has been solved. Quantities of fluid properties and boundary conditions in all cases were considered in such a way that by shifting each parameter, other dimensionless numbers remain constant.

\subsection{Rayleigh Number}

Considering the aim to be investigation of turbulence, the range of Rayleigh number between 109 and 1012 has been studied. Dimensionless shear stress is defined as follows [5].

$\tau_{\omega}^{*}=\frac{V_{B}}{g \beta \Delta T}\left(\frac{\partial u}{\partial x}\right)_{x=L}$

Fixed values for other dimensionless numbers in this mode are selected in the following form:

$R e_{B}={ }_{5}, \operatorname{Pr}={ }_{0.72,}$ Stef $=_{200,} H / L={ }_{4}, d / L=0.008$

In Fig. [3] The average dimensionless shear stress changes on a heated screen based on Rayleigh number is shown. According the lower Rayleigh values, Rayleigh highly affects shear stress, whereas with increased Rayleigh, the amount of this effect decreases which is consistent with in other papers [7-5]. In high Rayleigh, the effects Rayleigh on the stress, remains constant for highly increased Rayleigh. 


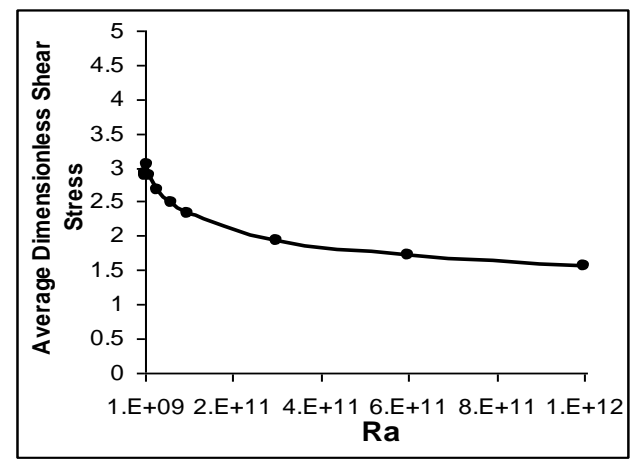

Figure 3. The effect of Rayleigh number on the average dimensionless shear stress along the wall containing blowing

Fig. [4] Indicates the relative turbulent viscosity variations in viscosity of the fluid with Rayleigh number. It can be observed that in low Rayleigh numbers, the only turbulent flow is at the end the channel in hot wall blowing wall, which reaches the maximum level in the external opening. However, with the increase in the Rayleigh number, turbulent flow occurs earlier and in addition to the hot wall, the boundary layer which was caused by the isolated wall is also turbulent. In Rayleigh number $10^{12}$, almost the entire channel is turbulent in which, the turbulent viscosity is approximately 300 times more than fluid viscosity. This turbulence has a significant effect on the heat transfer and wall shear stress.

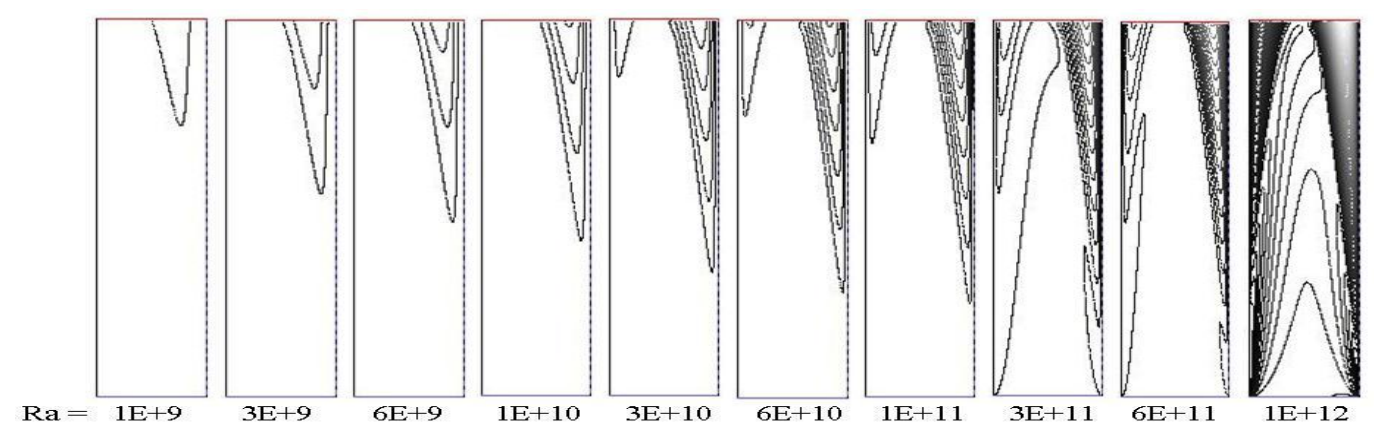

Figure 4. The effect of Rayleigh changes on Turbulent Viscosity level with fluid viscosity in the channel

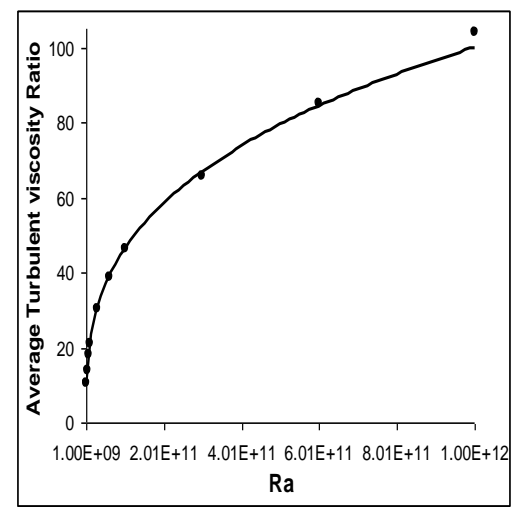

Figure 5. The effect of Rayleigh on the average turbulent viscosity ratio with fluid viscosity in the outlet 
In Fig. [5], the average relative turbulent viscosity against the Rayleigh number variations is drawn, which indicates that the turbulent viscosity dependence on Rayleigh number is an exponential dependence with negative concavity. To define the reference velocity and dimensionless velocity, we applied the definition of reference velocity in free convection on a vertical plate. For such a plate, the reference velocity is defined as $\frac{\alpha}{H} R a^{\frac{1}{2}}$ [24]. For the investigated problem, the output velocity caused by blown discharges, the value will also be added in the channel output. Thus the sequence of reference velocity and dimensionless velocity are defined as:

$$
\begin{aligned}
& V_{\text {ref }}=\left(\frac{\alpha}{H} R a_{H}^{\frac{1}{2}}+\frac{H}{2 L} V_{B}\right) \\
& V^{*}=V \times\left(\frac{\alpha}{H} R a_{H}^{\frac{1}{2}}+\frac{H}{2 L} V_{B}\right)^{-1}
\end{aligned}
$$

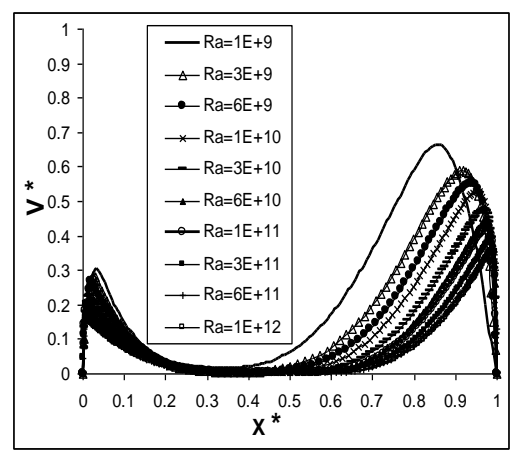

Figure 6. The effect of Rayleigh number on the dimensionless velocity in the outlet channel

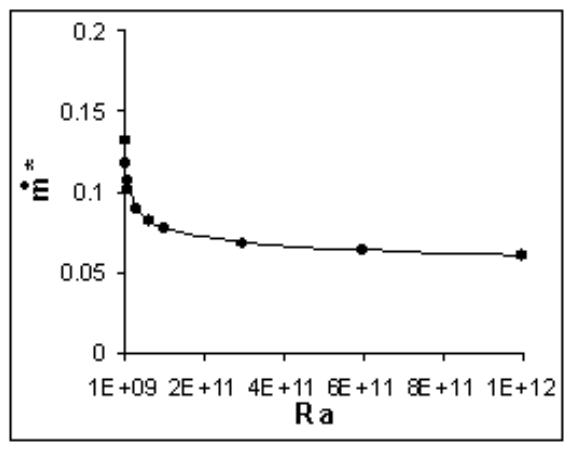

Figure 7. The effect of Rayleigh number on dimensionless mass flow rate suctioned from the channel input

Fig. [6] Displays dimensionless velocity profile variation as the Rayleigh changes. It can be seen that the overall pattern of the diagram remains constant with the increase in Rayleigh, but the impact of Rayleigh on velocity decreases which is considered in shear stress. Also, with the increase of Rayleigh, the relative thickness of the boundary layer reduces and the flow gets closer to the wall. In order to make the input suctioned mass discharge dimensionless, the first statement of the velocity statement is applied. Thus, the suctioned dimensionless mass discharge will be as follows: 


$$
\dot{m}^{*}=\dot{m} \times\left(\frac{\rho_{0} L \alpha}{H} R a_{H}^{\frac{1}{2}}\right)^{-1}
$$

In which $\rho_{0}$ is the density of suctioned fluid in the input temperature. Figure (7) shows the relation between the dimensionless mass suctioned from the channel input with Rayleigh number. It can be perceived that the slope of the diagram is very high whereas it decreases with the increase in Rayleigh number. Figure (8) shows that as the Rayleigh increases, the role of blowing and radiation in heat transfer gradually decreases to $15 \%$ and $5 \%$, while a $20 \%$ increase is observed for convection heat transfer

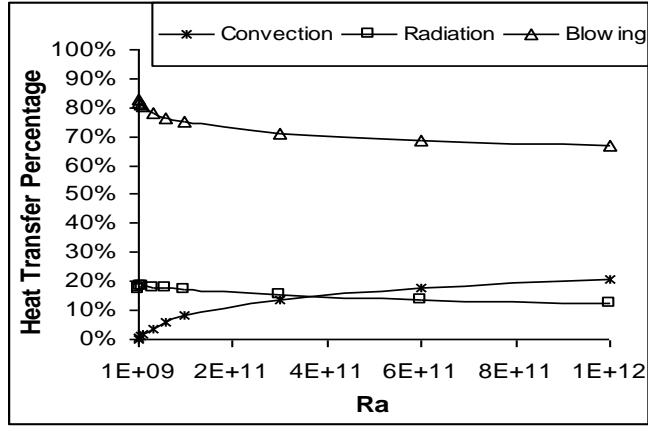

Figure 8: the effect of Rayleigh number on the percentages of heat transfer

\subsection{Blowing Reynolds Number}

In order to investigate the effects of Reynolds number, a range of Reynolds number between 0 and 15 were studied. Fixed values for other dimensionless numbers were selected this way:

$$
R e_{B}=1010, \operatorname{Pr}=_{0.72}, \text { Stef }=_{200}, H / L=4, d / L=0.008
$$

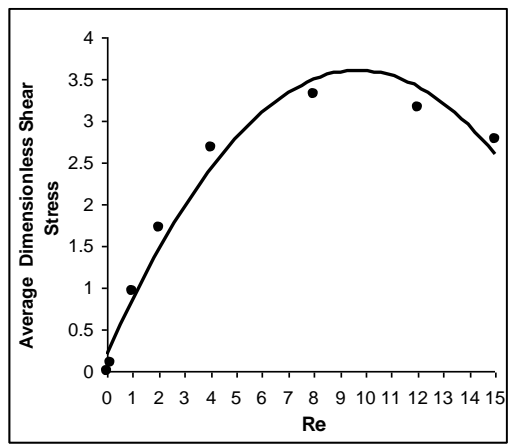

Figure 9: the effect of Reynolds number on the average dimensionless shear stress along the wall containing blowing

Figure (9) shows the average shear stress on the wall with Reynolds number variations which changes in parabolic function with negative concavity. Considering the different states, some interesting results can be achieved. First, we consider that Reynolds has a linear relation with velocity, and the dimensionless shear stress has a direct correlation velocity and the shear stress of the wall. If the increase in Reynolds is merely the results of increase in velocity, then the negative concave shows a decrease in the wall shear stress with the increase of velocity. The result is due to the fact that the blown warm fluid pushes back the flowing fluid in the channel 
and blocks the creation of shear stress between the fluid layers in the channel and the well. Also, as the velocity and consequently the Reynolds increases, the impact of the pushing back increases too. If the increase in Reynolds is associated with a constant speed, it can be concluded that increase in Reynolds (increase in density or decrease in viscosity diameter) can increases the shear stress. However, this trend continues as long as the boundary layers of the two walls did not meet. In Reynolds number more than 8 , in which the boundary layers meet, the impact of the increase in Reynolds (with constant velocity), becomes reverse. Figure (10) shows the effects of Reynolds variation on the turbulent viscosity ratio to fluid viscosity in the channel.

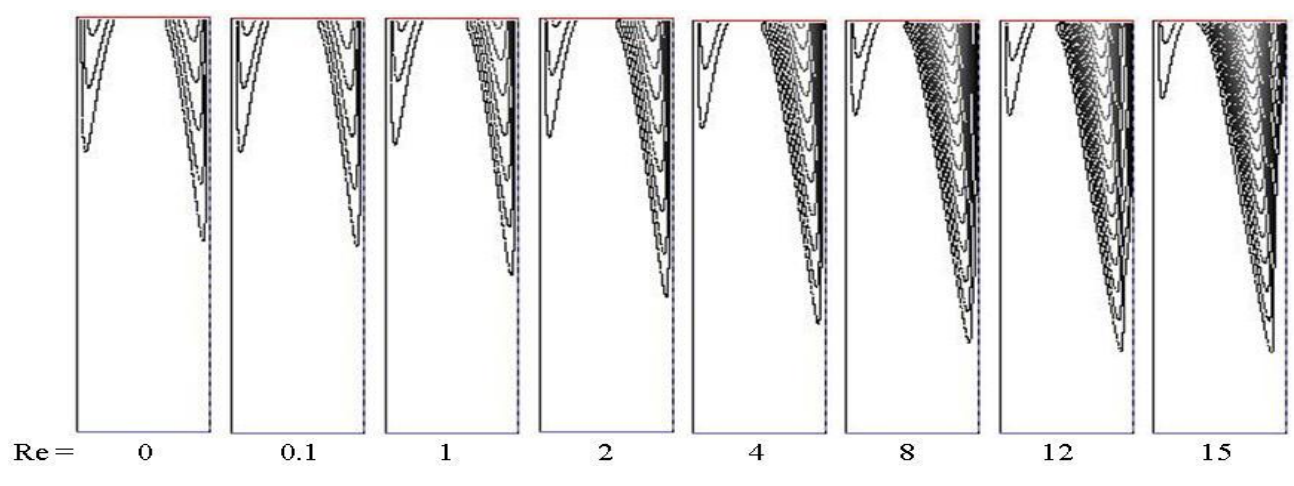

Figure 10 : The effects of Reynolds variation on the turbulent viscosity ratio to fluid viscosity in the channel.

In Fig. (11) The variations in output dimensionless velocity is shown with respect to the variations in Reynolds number. Although the blowing Reynolds variations do not have a significant impact on the boundary layer of the insulated wall, it causes the boundary layer of the heated blowing wall to extend strongly. Increase in the blowing Reynolds not only augments the amount of diagram's quantity curve, it will also increase the distance of maximum point of the graph's curve from the right part of wall in which Rayleigh number growths inversely.

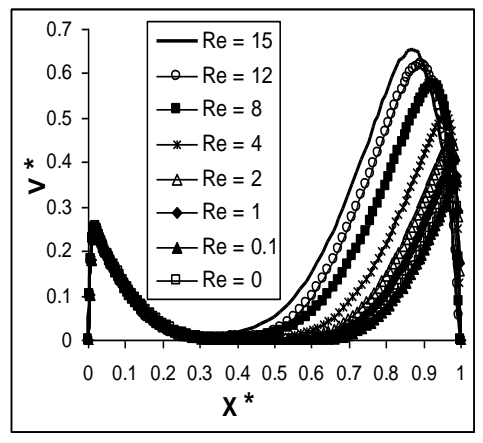

Figure 11. : The effect of Reynolds on the dimensionless velocity in the outlet channel.

Fig. (12) Demonstrates that Increase in Reynolds Number can also augment the negative concavity of the dimensionless mass flow of the fluid suction from the inlet opening. At lower Reynolds numbers range, there is higher effect of increasing blowing Reynolds on the suctioned mass flow, but its impact reduces gradually. The reason of mass flow is evident according to what has been already mentioned in shear stress variation. In figure (13), it is clear that in non-blowing mode, the blowing is 55\% through radiation heat transfer and the rest is done by convection. However, as the Reynolds increases, blowing play the most important role in heat transfer. 


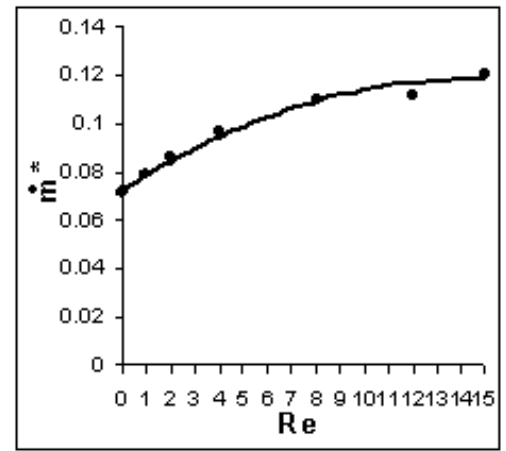

Figure 12. : The effect of Reynolds on the dimensionless mass flow suctioned from the channel input

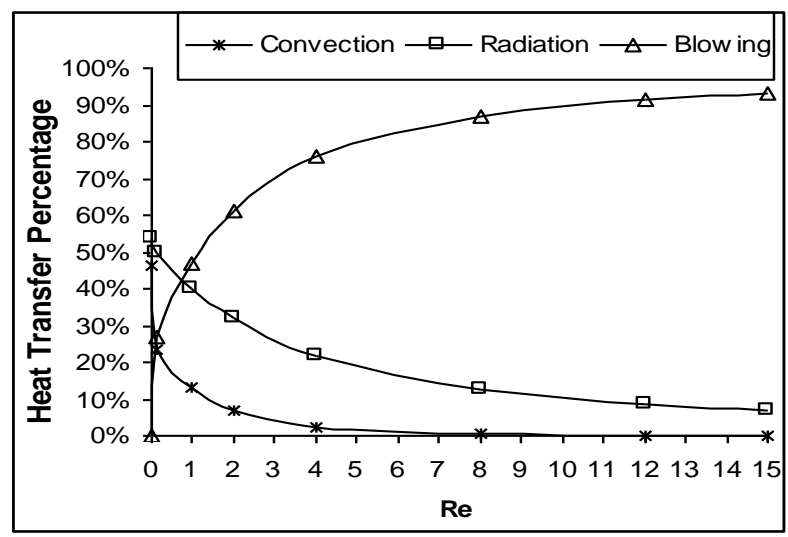

Figure 13: the effect of Reynolds number on the percentage of heat transfer components

\subsection{Stephen Numbers}

In order to study the radiation parameters, a range of Stephen numbers from 0 to 250 has been investigated. The fixed values for other dimensionless numbers in this state were selected by this method:

$$
R e_{B}={ }_{1010,} \operatorname{Re}_{B}={ }_{5}, \operatorname{Pr}={ }_{0.72}, H / L={ }_{4}, d / L=0.008
$$

Figs (14) show that the radiation parameters (Stephen number) had an insignificant impact on the average dimensionless wall shear stress with blowing, while an increase in Stephen number causes a rise in the dimensionless shear stress of the insulated wall with parabolic function.

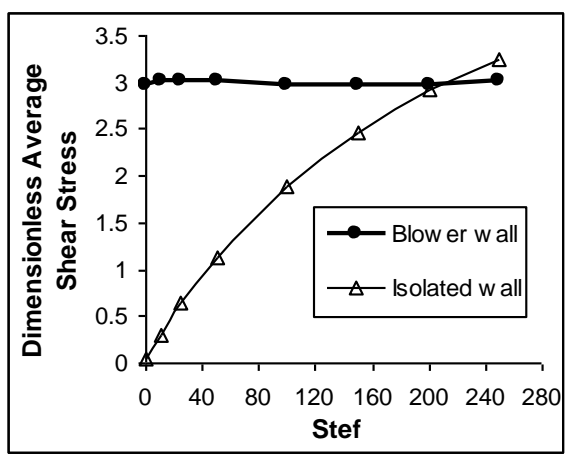


Figure 14: The effect of Stefan number on the average dimensionless shear stress along the wall containing blowing and isolation

Figs. (15) show the relative turbulent output viscosity in which the pattern and values are constant in the right part of the graph (except for the radiation less state) whereas augmentation of radiation parameters causes' growth in turbulence of the channel's left part.

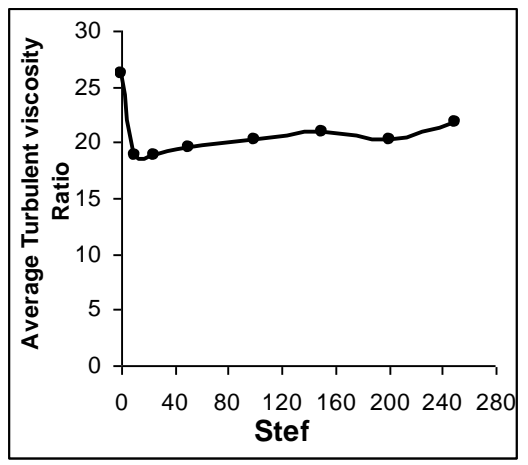

Figure 15: the effect of Stefan number on the average ratio of turbulent viscosity to the fluid viscosity in the outlet channel.

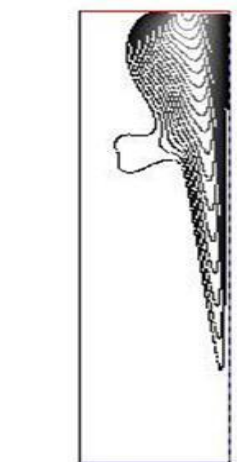

Stef $=0$

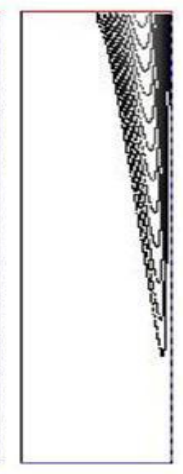

10

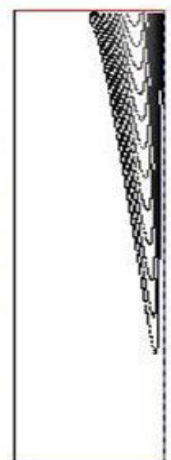

25

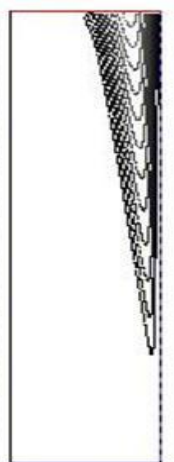

50

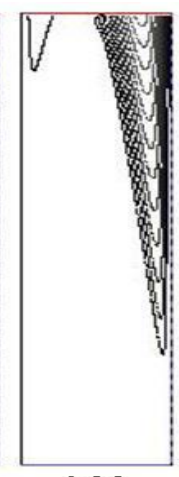

100

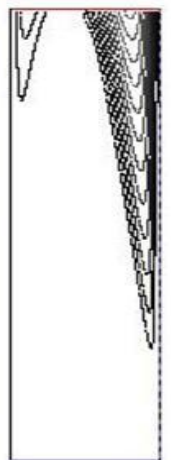

150

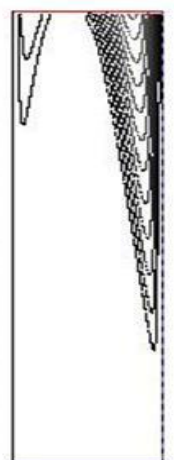

200

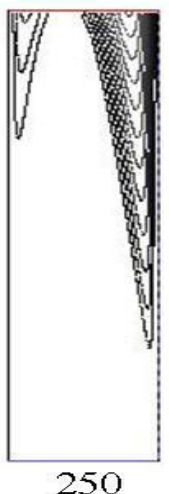

Figure 16: he effect of Stefan number variability on the ratio of turbulent viscosity to the viscosity of the fluid in the channel

Fig. (16) Also illustrates that, except for the Stephen number in Zero mode, radiation does not have a significant impact on the turbulent flow. In non-radiation mode, there is a return flow in the left part of channel which causes graph pattern to change completely. However, despite the radiation, there is an upward flow in the left part of the graph which blocks the return flow.

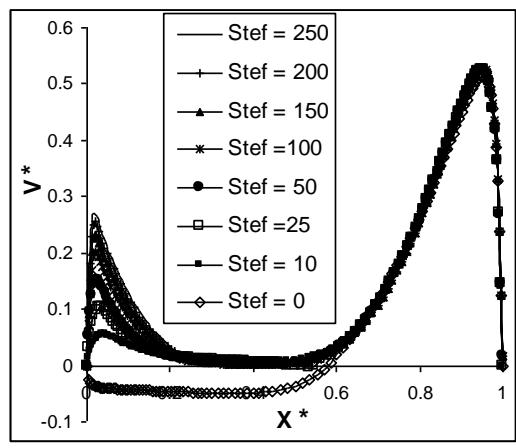


Figure 17: the effect of Stephan number on the dimensionless temperature in the outlet channel.

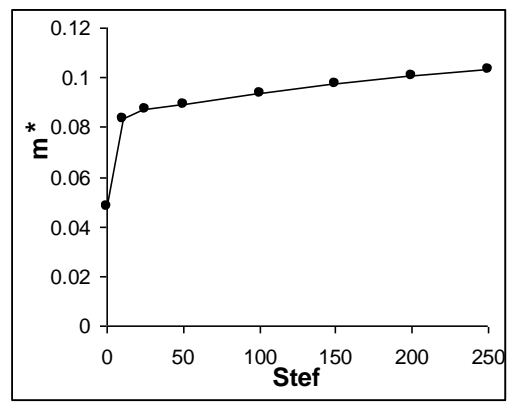

Figure 18: the effect of Stephan number on the dimensionless mass flow suctioned from the input channel

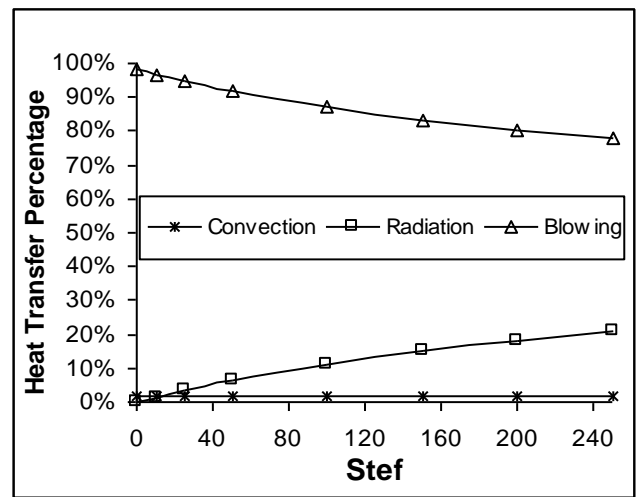

Figure 19: the effect of Stefan number on the percentage of heat transfer components.

Fig. (17) Shows the output velocity of dimensionless profile in channel, which is drawn for different Stephen numbers. In general, it can be observed that decrease in Stephen number can causes decline in values of the left part, but does not have any effect on the boundary layer of the right part in channel. The negative values of velocity in non-radiation mode indicate existence of return flow. Fig. (18) Shows that despite the line radiation, the amount of dimensionless mass flow which is suctioned from the input opening changes with the Stephen number. The reason for decrease in the graph values in non-radiation mode can be the existence the return flow. In figure (19), it is illustrated that as the Stefan number increases, the impact of radiation in transfer increase by $20 \%$ and the impact of blowing decreases $20 \%$, while the impact of convection is insignificant and remains constant.

\section{CONCLUSION}

In this paper, the effect of radiation and blowing on free convection of fluid flow parameters was studied in a vertical channel. The results clearly demonstrate that the radiation process acts independent of blowing and free convection on the radiation wall. Blowing from wall has a double effect on shear stress of the radiation wall which led to cause increase in lower Reynolds numbers, and a decrease in shear stress in higher Reynolds numbers. Also, blowing increases the turbulence and the flow rate suctioned from input of the channel. In addition, effect of the Rayleigh number is higher on flow in lower Rayleigh number and the effect decreases as the Rayleigh number goes up.. 


\section{REFERENCES}

[1] Tsuji, T., Nagano, Y., "Characteristics of a Turbulent Natural Convection Boundary Layer along a Vertical Flat Plate", Int. J. Heat Mass Transfer, vol.31, pp. 1723-1734, (1988).

[2] Tsuji, T., Nagano, Y., "Turbulence measurements in natural convection boundary layer along a vertical flat plate", Int. J. Heat Mass Transfer, Vol. 31, pp. 2101-2111, (1988).

[3] Gupta, A. S., Misra, J.C., Reza, M., "Effects of suction or blowing on the velocity and temperature distribution in the flow past a porous flat plate of a power-law fluid", J. of Fluid Dynamics Research, Vol. 32, pp. 283-294, (2003).

[4] Eichhorn, R., "The effect of mass transfer on free convection", J. of Heat Transfer, Vol. 82, pp. 260-263, (1960).

[5] Bellettre, J., Bataille, F., Rodet, J. C., Lallemand, A., "Thermal behaviour of porous plates subjected to air blowing", AIAA J. Thermophys. Heat Transfer 14 (4) (2000) 523-532.

[6] Whitten, D.G., Moffat, R.J., Kays, W.M., "Heat transfer to a turbulent boundary layer with nonuniform blowing and surface temperature", in: 4th Int. Heat Transfer Conf., 1970.

[7] Bellettre, J., Bataille, F., Lallemand, A., "A new approach for the study of turbulent boundary layers with blowing", Int. J. Heat Mass Transfer 42 (15) (1999) 2905-2920. [2] Tsuji, T., Nagano, Y., "Turbulence measurements in natural convection boundary layer along a vertical flat plate", Int. J. Heat Mass Transfer, Vol. 31, pp. 2101-2111, (1988).

[8] Brillant, G., Bataille, F., Ducros, F., "Large-eddy simulation of a turbulent boundary layer with blowing", Theor. Comput. Fluid Dyn. 17 (2004) 433-443.

[9] Mathelin, L., Bataille, F., Lallemand, A., "Near wake of a circular cylinder submitted to blowing - I boundary layers evolution", Int. J. Heat Mass Transfer 44 (2001) 3701-3708.

[10] Mathelin, L., Bataille, F., Lallemand, A., "Near wake of a circular cylinder submitted to blowing - II impact on the dynamics", Int. J. Heat Mass Transfer 44 (2001) 3701-3708.

[11] N. Shima, Prediction of turbulent boundary layers with a secondmoment closure: Part i - effects of periodic pressure gradient, wall transpiration and free-stream turbulence, Trans. ASME J. Fluid Eng. 115 (1) (1993) 56-63.

[12] A.P. Silva-Freire, D.O.A. Cruz, C.C. Pellegrini, Velocity and temperature distributions in compressible turbulent boundary layers with heat and mass transfer, Int. J. Heat Mass Transfer 38 (13) (1995) 2507-2515.

[13] Merkin, J. H., "Free Convection with Blowing and Suction", Int. J. Heat Mass Transfer, Vol. 15, pp. 989-999, (1972).

[14] Vedhanayagam, M., Altenkirch R. A., Eichhorn, R., "A Transformation of the Boundary Layer Equations for Free Convection Past a Vertical Flat Plate with Arbitrary Blowing and Wall Temperature Variations", Int. J. Heat Mass Transfer, Vol.23, pp. 1286-1288, (1980).

[15] Brouwers, H. J. H., "The Film Model Applied to Free Convection Over a Vertical Plate with Blowing or Suction", Int. J. Heat Mass Transfer, Vol. 35, No. 7, pp. 1841-1844, (1992).

[16] Cheng, X., and Muller, U., "Turbulent natural convection coupled with thermal radiation in large vertical channels with asymmetric heating", Int. J. Heat Mass Transfer, Vol. 41, No. 12, pp. 1681-1692, (1998).

[17] Dong, Y.-H., Lu, X.-Y., "Large eddy simulation of a thermally stratified turbulent channel flow with temperature oscillation on the wall", International Journal of Heat and Mass Transfer 47, 2109-2122, (2004).

[18] Wang, W.P., Pletcher, R.H., 1996. On the large eddy simulation of a turbulent channel flow with significant heat transfer. Physics of Fluid 8 (12), 3354-3366.

[19] Murata, A., Mochizuki, S., 2004a. Large eddy simulation of turbulent heat transfer in a rotating two-pass smooth square channel with sharp 180_turns. International Journal of Heat and Mass Transfer 47, 683-698. 
[20] Murata, A., Mochizuki, S., 2004b. Effect of rib orientation and channel rotation on turbulent heat transfer in a two-pass square channel with sharp 180_ turns investigated by using large eddy simulation. International Journal of Heat and Mass Transfer 47, 2599-2618.

[21] Lee, J.S., Xu, W., Pletcher, H., 2004. Large eddy simulation of heated vertical annular pipe flow in fully developed turbulent mixed convection. International Journal of Heat and Mass Transfer 47, 437-446.

[22] Chatelain, A., Ducros, F., Métais, O., 2004. LES of turbulent heat transfer: proper

[23] Abd El-Aziz, M., "Thermal-diffusion and diffusion-thermo effects on combined heat and mass transfer by hydromagnetic three-dimensional free convection over a permeable stretching surface with radiation", Physics Letters A, Vol. 372, pp. 269-276, (2008).

[24] Bejan, A., "Convection Heat Transfer", Third Edition, John Wiley \& Sons, New Jersey, (2004).

Authors

ali yari was born in Septembr 21nd 1983 in Qaemshahr, Mazandaran. He received his Ms. degree in mechanical engineering (Energy Conversion) from Shiraz University, Shiraz, Iran. He also received his BSc degree in thermal fluids from in thermal fluids from BabolNoshirvani University of Technology, Babol, Mazandaran.He did his military service in Education as a Soldier teacher from 2010 to 2012. He is currently a university lecturer in Universities in Mazandaran, Iran

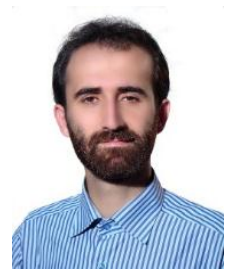

Samak. Hosseinzadeh was born in August 22nd 1985 in Sari,Mazandaran. He is PhD student of mechanical field (energy conversion) at the Department of Mechanical Engineering, West Tehran Branch, Islamic Azad University, Tehran, Iran. He received his Ms. degree in mechanical engineering (Energy Conversion) from South Tehran Branch in 2010. He also received his BSc degree in thermal fluids from Islamic Azad University Sari Branch, Mazandaran in 2007.He did his military service in Manufacturing, Mining and Trade organization as an industry expert from 2010 to 2012. He is currently a university lecturer in Islamic Azad

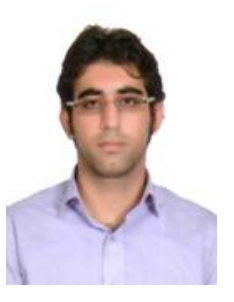
Universities in Mazandaran, Iran. The teaching courses included Mechanical technology, Automotive and Machinery, Power Plant Technology, Installation Technology, Chemical Industry, Architecture, Power Electronics, Project Management and Executive Management. He also was a Technical Office Manager (Head Office) in Nogostaran Construction Installation Company in Tehran from 2007 to 2011. He is a member of Iran Construction Engineering organization.

Meghdad Rahimi Galogahi was born in Monday, July 2, 1979 in Behshahr, Mazandaran. He received his MS. degree in mechanical engineering, Energy conversion from Babol,Noshirvani University of Technology, Babol, Mazandaran in 2005 . He also received his BSc degree in thermal fluids from Babol,Noshirvani University of Technology, Babol, Mazandaran. He is currently faculty member of Islamic Azad University, Behshahr, Mazandaran, Iran. He is also the Managing Director and employer of produced Payab cartons. He is a member of Iran

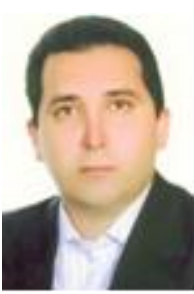
Construction Engineering organization. 